\title{
Strong phenomenal wholes are associated with fast "same" and slow "different" responses and superior overall performance
}

\author{
DONALD L. KING \\ Howard University, Washington, D.C.
}

\begin{abstract}
"Same" and "different" responses to stimuli that result in strong and weak single phenomenal wholes were investigated. Present and also previous results indicate that strong phenomenal wholes tend to be associated with a fast "same" and slow "different" response pattern. This result suggests that the parts of strong phenomenal wholes are perceived as more similar (e.g., because it should be easier to respond "same" to more similar stimuli). This suggestion is consistent with results based on other measures of perceived similarity. Present and also previous results additionally indicate that strong phenomenal wholes are frequently associated with superior overall ("same" plus "different") performance. This result is consistent with evidence that the identification of simultaneously occurring multiple targets improves when they belong to the same phenomenal whole. The present results also hint that attention can affect whether two or three objects form the effective (functional) whole and support the assimilation explanation of the MüllerLyer illusion.
\end{abstract}

Speeded "same" and "different" responses to figural stimuli have been investigated. Theoretical interests have involved serial and parallel processing (Beller, 1970; Donderi \& Zelnicker, 1969; Hawkins \& Shigley, 1972; Krueger, 1973), features (diagnostics) that affect performance (Fox, 1975; Richards, 1978), and the normalization of irrelevant dimensions (Dixon \& Just, 1978). Nevertheless, past and present research supports two other interpretations-and both involve phenomenal wholes (gestalts). Richards (1978) probably came closest to this involvement by maintaining that whole-display structural information was utilized.

\section{Associations Between Wholes and an FSSD Pattern}

There is evidence that strong phenomenal wholes are usually associated with fast "same" and slow "different" (FSSD) responses. One of Dixon and Just's (1978) results is illustrative. The stimuli were pairs of adjacent ellipses. When the task was to respond "same" to ellipses of the same height, an increase in the disparity of the widths of these ellipses increased "same" RTs but did not noticeably affect "different" RTs. The pairs that were identical in width presumably formed the strongest wholes, in accord with the gestalt principle of similarity

This research was supported by Alcohol Drug Abuse and Mental Health Administration (NIMH) Grant 5-T-34-MH16580. The author wishes to thank Lenese Herbert, Donya Norton, and Mary Ross for help with data collection and analysis. Experiment 1 was reported at the Psychonomic Society meeting in San Antonio, November 1984. Experiments 2 and 3 were reported at the Eastern Psychological Association meeting in Boston, 1985. Please send requests for reprints to Donald L. King, Department of Psychology, Howard University, 525 Bryant St., N.W., Washington, DC 20059 (also see Dixon \& Just's Figure 1). Therefore, strong phenomenal wholes were associated with the FSSD pattern.

The FSSD pattern is indicated by a type of response $X$ strength of whole interaction, as in the Dixon and Just (1978) study. Main effects for both "same" and "different" responses in opposite directions are not required, because additional factors may affect "same" and "different" responses in the same direction. In addition, the FSSD pattern is sometimes considered to occur when a significant interaction is not reported, frequently because "same" and "different" responses were analyzed separately, as in the Dixon and Just study once again.

Stimuli that were similar in size, shape, and color also resulted in the FSSD pattern (Hawkins \& Shigley, 1972), as did stimuli that were similar by virtue of having common parts (Krueger, 1973). In addition, letters with bilateral and overall symmetry (Fox, 1975), bilaterally symmetric forms made from a number of letters (Millspaugh, 1978), and collinear and parallel lines (Fox, 1975) also resulted in the FSSD pattern. Also, Richards (1978) compared symmetric and asymmetric stimuli using letters and dot patterns and obtained the FSSD pattern in some experiments. Symmetry, colinearity, and parallelism are all types of similarity. Similar stimuli generally form strong phenomenal wholes. Furthermore, drawings in the above studies suggest that the more similar stimuli form stronger phenomenal wholes. In conclusion, these studies found that strong phenomenal wholes were usually associated with the FSSD pattern.

In another experiment (Beller, 1970), the stimuli included two, four, and eight identical letters in a row. When the letters were physically identical or when one letter differed from the remaining letters, an increase in 
the number of letters did not increase "same" RTs but did increase "different" RTs. An increase in the number of letters should have also increased the strength of a phenomenal whole that approximates a horizontal line. Therefore, strong phenomenal wholes were associated with the FSSD pattern. The letters in an experiment by Krueger and Shapiro (1981) were arranged in a circle. An increase in the number of letters also resulted in the FSSD pattern. The increase in the number of letters should have also increased the strength of a circle-like whole. In both these experiments, multiple different letters resulted in fast "different" responses, presumably because the occurrence of these multiple differences more quickly satisfied the decision criterion for "different." Donderi and Zelnicker (1969) also found that an increase in the number of objects resulted in the FSSD pattern. This increase probably also increased the impression of a clump (whole) of objects.

More similar objects do not always result in the FSSD pattern. Richards (1978) did not obtain this result for some comparisons between symmetric and asymmetric stimuli. In addition, Richards did not find that stimuli with the same tilt resulted in the FSSD pattern. However, it is doubtful that the same tilt stimuli resulted in stronger phenomenal wholes (see his Figure 1). Egeth, Brownell, and Geoffrion (1976) did not obtain the FSSD pattern in one experiment with symmetric and asymmetric letters (in other experiments, only "same" RTs were measured). More proximal objects should also result in stronger phenomenal wholes. However, overlapping letters and pairs of more proximal letters did not result in the FSSD pattern (Fox, 1975 and Chignell \& Krueger, 1984, respectively). The FSSD pattern can occur only when selective attention to targets is incomplete. Therefore, the inconsistent evidence might be due to good selective attention.

Physical similarity is not consistently associated with the FSSD pattern, because an increase in the number of objects also results in this pattern. In addition, an effect of overall display size was controlled for in the studies by Fox (1975), Hawkins and Shigley (1972), Krueger (1973), Millspaugh (1978), and Richards (1978). Also, an effect of the average distance between objects was controlled for in the studies by Beller (1970), Fox (1975), Hawkins and Shigley (1972), Krueger (1973), Millspaugh (1978), and Richards (1978). Therefore, strong phenomenal wholes are associated with the FSSD pattern more consistently than is a particular type of feature.

Perceived similarity. The FSSD pattern is considered to indicate high perceived similarity. One reason is that it should be easier to decide that perceptually more similar stimuli are identical in one parameter and harder to indicate that perceptually more similar stimuli are different in this parameter. In addition, the ability of anchor (extreme) stimuli to impair the identification of more similar stimuli (Gravetter \& Lockhead, 1973; Pollack, 1952) can be considered to be due to an increase in the perceived similarity of the more similar stimuli. Therefore, evidence that three types of anchor-like contextual effects resulted in the FSSD pattern (King, 1983; King \& Atef-vahid, 1986, Experiments 2-4 and 5) also implies that the FSSD pattern indicates high perceived similarity. As mentioned earlier, strong phenomenal wholes are associated with the FSSD pattern. Therefore, strong phenomenal wholes are associated with an increase in the perceived similarity of their parts (i.e., assimilation). Evidence based on other measures of perceived similarity supports this conclusion (King, in press; this article's General Discussion).

The possible influence of attention. In an experiment by King and Atef-vahid (1986), three disks were equally spaced around an imaginary circular line. The weaker whole (or the stronger two whole) stimuli consisted of two small disks and one extremely large (i.e., anchor) disk. The stronger whole (or the weaker two whole) stimuli consisted of the identical two small disks and one fairly (not extremely) large disk. The task was to respond "same" when the two small disks were the same in size and to respond "different" when all three disks were different in size. The weak whole stimuli resulted in the FSSD pattern. This finding suggests that the two similar disks were perceived as more similar in the context of an extreme stimulus. Therefore, it is an anchor-like result. Nevertheless, it contrasts with the conclusion that strong phenomenal wholes are associated with the FSSD pattern.

The large disk was always different from the two small disks. Therefore, it was logically irrelevant. Consequently, the subjects should have attempted to attend to only the two small disks. When we simultaneously attend to two objects, we seem to experience them as a single whole to a greater extent. Furthermore, the extremely large disk might have made the two small disks form a stronger whole than the fairly large disk, much as a third dot in an extreme spatial position makes two more proximal dots group more strongly. Granted these assumptions, the stronger phenomenal whole was associated with the FSSD pattern.

Therefore, attention to two similar stimuli might have overridden the influence of an extremely dissimilar stimulus. If so, features could not have directly (immediately) resulted in the FSSD pattern. Furthermore, attention could have altered the phenomenal wholes that occurred, as was mentioned. Consequently, it is possible that a factor closely related to the strength of phenomenal wholes, rather than to features, was directly responsible for the FSSD pattern.

\section{Associations Between Wholes and Overall Performance}

The following studies found that objects with more physically similar parts resulted in faster overall ("same" plus "different") responses: Dixon and Just (1978), Fox (1975), Hawkins and Shigley (1972), Krueger (1973), and Richards (1978). Furthermore, these results were not due to speed-accuracy tradeoffs. Stimuli with more physically similar parts should result in stronger phenomenal wholes. Therefore, strong phenomenal wholes were associated with superior overall performance. Identification task per- 
formance also improves when two or more targets (parts) belong to the same phenomenal whole (see General Discussion).

The following studies did not find that strong phenomenal wholes were associated with superior overall "same"'-"different" performance: Beller (1970), Chignell and Krueger (1984), Donderi and Zelnicker (1969), Egeth et al. (1976), Krueger and Shapiro (1981), and Millspaugh (1978). In the Beller, Donderi and Zelnicker, and Krueger and Shapiro studies, the stronger phenomenal wholes contained more objects. Therefore, a tendency to serially process objects individually would account for their negative (actually, reverse) evidence. It is well known that proximal stimuli are difficult to discriminate between, so the Chignell and Krueger negative evidence is also understandable. However, the possible attention-derived strong phenomenal wholes of King and Atef-vahid (1986) were also associated with poor overall performance. In General Discussion, the negative evidence is considered further.

\section{EXPERIMENT 1}

The studies that have been considered did not seriously entertain a role for phenomenal wholes. Therefore, the purpose of Experiment 1 was to determine if strong phenomenal wholes would be associated with the FSSD pattern and superior overall performance when stimuli were selected on the basis of the strength of the phenomenal wholes that they resulted in. The strong whole stimuli consisted of three equally spaced disks in a row. These disks readily result in a line-like (row-like) single phenomenal whole. The weak whole stimuli were formed by moving the middle disk to the left or right. These stimuli seem to more readily form two phenomenal wholes: the two relatively proximal disks form one whole, and the spatially extreme disk forms a second (singleton) whole.

These strong and weak whole stimuli were selected for several purposes. The experiment in which weak phenomenal wholes were associated with the FSSD pattern (King \& Atef-vahid, 1986) also employed an extreme third disk, albeit one that was extreme in size. The explanation for this discrepancy involved the assumption that the instructions directed attention to only the two small disks. In the present experiment, attention should be directed to all three disks. Therefore, the strength of the phenomenal whole formed by all three disks should be predictive. Consequently, if the equally spaced disks (i.e., strong whole stimuli) resulted in the FSSD pattern, an influence of attention would be suggested.

Recall that an increase in the number of objects in a row increases the strength of a line-like phenomenal whole and also results in the FSSD pattern (Beller, 1970). Therefore, with the present stimuli, it could be determined if the line-like strong whole type of stimulus would be as- sociated with the FSSD pattern when the parts differed in spacing rather than in number. Finally, with the present stimuli, the overall distance and the average interpart distance of the strong and weak whole stimuli were equated.

The procedures employed in the other experiment with an extreme third disk (King \& Atef-vahid, 1986) were followed closely in order to determine if the reverse result could be obtained with essentially the same procedures. Therefore, blocks of trials with one type of stimulus (strong whole or weak whole) were followed unexpectedly by single trials with the second type of stimulus and vice versa. The original purpose of this procedure was to determine whether responding was due to stimuli that occurred on a current trial or to memory for stimuli that occurred on preceding trials.

\section{Method}

Stimuli. Figure 1 shows the equally spaced (strong whole) and irregular (weak whole) stimuli that were used. The $\mathbf{S}$ (small) disks were $5 \mathrm{~mm}$ in diameter, and the $\mathrm{T}$ (tiny) disks were $3 \mathrm{~mm}$ in diameter. The centers of the left and right disks were separated by $25 \mathrm{~mm}$. The center of the middle disk of the equally spaced stimuli was halfway between the centers of the left and right disks, whereas the center of the middle disk of the irregular stimuli was $7.5 \mathrm{~mm}$ away from the center of the closer of the two end disks. The disks were obtained from Letraset Sheet 2453. The stimuli were centered on white cards.

The set of stimuli was incomplete in that stimuli with two and with three T disks were not used. Nevertheless, the present results closely resemble the results of Experiments 2 and 3.

Experimental design and trials. One group of subjects was instructed to respond "same" when all the disks were the same in size, and the other group was instructed to respond "different"
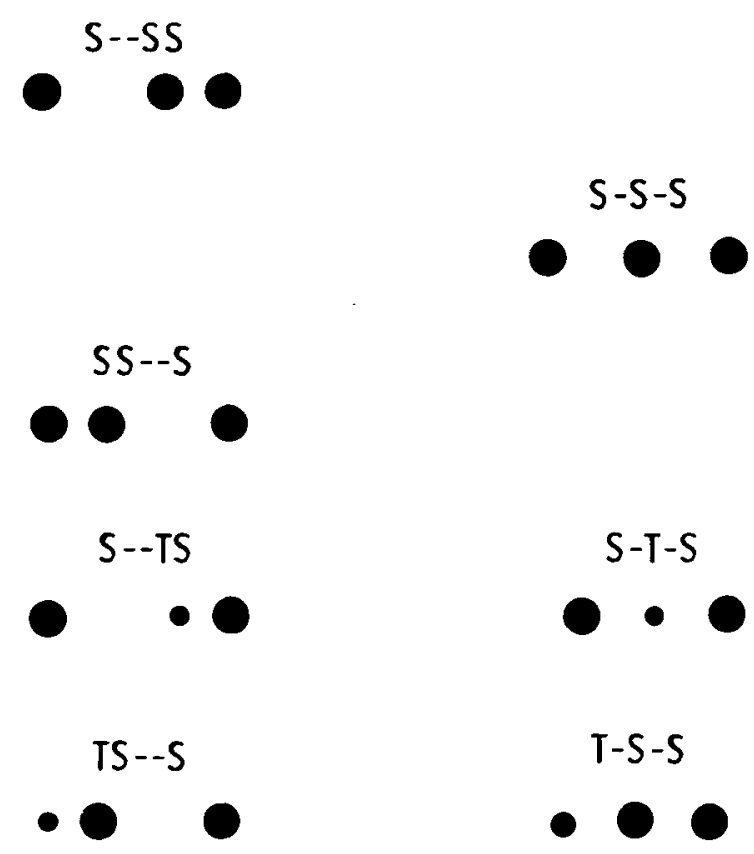

Figure 1. The stimuli for Experiment 1. 
when one of the disks was different in size from the other two. Therefore, the occurrence of a difference between any two disks required a "different" response, and thus all three disks should have been attended to.

All subjects received an initial five sequences of trials (Stage 1) and a subsequent five sequences of trials (Stage 2). For half of both the "same" and "different" response groups, each sequence of Stage 1 consisted of a block of trials with equally spaced stimuli followed by a single unexpected trial with an irregular stimulus. The trial with the irregular stimulus was unexpected, because the number of trials in each block was variable, ranging from 5 to 12 , and because there was no other way to predict when the irregular stimulus would occur. The subsequent five sequences of blocks (Stage 2) followed immediately. For this stage, the irregular stimuli occurred in the blocks, and the equally spaced stimuli occurred on the unexpected trials.

The other half of both the "same" and "different" response groups received the equally spaced and irregular stimuli in the reverse order. That is, the blocks of trials of Stage 1 were with irregular stimuli, and so on.

The rest of the trial procedures also followed the earlier experiments (King, 1983; King \& Atef-vahid, 1986, Experiment 5). The same and different stimuli within each block occurred in the same randomly determined order for all subjects. The same five blocks of trials were used for Stage 2, but they occurred in a different order. The trials for the equally-spaced-blocks-first and irregular-blocksfirst groups corresponded exactly. For example, the S- -TS stimulus of Figure 1 substituted for the S-T-S stimulus of Figure 1 and vice versa. In addition, either weak whole same stimulus (S--SS or SS--S) could substitute for the single strong whole same stimulus (S-S-S). The unexpected same stimuli occurred after the second and fourth blocks of Stage 1, and after the second and third blocks of Stage 2. Thus, the unexpected different stimuli occurred after the first, third, and fifth blocks of Stage 1, and after the first, fourth, and fifth blocks of Stage 2.

Apparatus, Procedure, and Subjects. An Iconix 6137 tachistoscope was used. The distance from the stimuli to the cornea was approximately $90 \mathrm{~cm}$. The visual angle subtended by the $25-\mathrm{mm}$ distance between the centers of the left and right disks was therefore about $1.6^{\circ}$.

The experimenter said "Ready" to initiate a trial. A visual warning stimulus, a $7.6 \times 12.7 \mathrm{~cm}$ gray square centered on a black background, was followed by $1 \mathrm{sec}$ of darkness and then one of the stimuli. This stimulus either remained on for $3 \mathrm{sec}$ or was terminated by the subject's response. The response was a press of a telegraph key with the dominant hand, and it terminated the clock. Timing was in milliseconds. The experimenter said "Correct" or "Wrong" after each response and also indicated the response time (RT) after correct responses.

There were five Howard University undergraduates in each of the four groups. Participation satisfied an introductory psychology course requirement.

\section{Results}

Response times. The analysis of variance (ANOVA) was a 2 (response: "same"' and "different") $\times 2$ (order: equally spaced stimuli in the blocks of Stage 1 first and irregular stimuli in the blocks of Stage 1 first) $\times 2$ (stimulus: equally spaced and irregular) $\times 2$ (trial: unexpected and block) factorial. The first two factors were betweensubject and the last two factors were within-subject.

In respect to "same" responses, there were two unexpected trials with same stimuli in each stage. The mean of the RTs to these two stimuli became an individual subject's entry for the ANOVA. There were two such entries, one for the equally spaced stimuli of one stage and one for the irregular stimuli of the other stage. The entries for the block trials were means based on the responses to only those blocks that immediately preceded the two unexpected same stimuli. The mean of the RTs to all the same stimuli in these two blocks became the entry for the equally spaced and irregular stimuli. Again, there were two such entries, one for the equally spaced stimuli of one stage and one for the irregular stimuli of the other stage. However, if a same stimulus immediately followed an unexpected trial (i.e., if it was the first stimulus in a block), the response to it was disregarded.

In respect to "different" responses, there were three unexpected trials with different stimuli in each stage. The mean of the RTs to these three stimuli was also an entry for the ANOVA, and again there were two such entries, one for the equally spaced stimuli of one stage and the second for the irregular stimuli of the other stage. The entries for the block trials were means based on the responses to only those blocks that immediately preceded the three unexpected different stimuli. Once more, there were two such entries, one for the equally spaced stimuli of one stage and the second for the irregular stimuli of the second stage. However, the responses to the first two different stimuli of the experiment were disregarded, and if a different stimulus immediately followed an unexpected trial (i.e., if it was the first stimulus in a block), the response to it was also disregarded.

Table 1 indicates the mean RTs for the 16 conditions. The interaction between the response and stimulus factors was significant $[F(1,16)=9.07, p<.01]$. Table 1 also indicates the means for this interaction. These means reveal that the interaction was due to the strong whole

Table 1

Mean RTs and Errors for Experiment 1

\begin{tabular}{|c|c|c|c|c|c|c|c|c|c|}
\hline \multirow[b]{3}{*}{ Trial } & \multirow[b]{3}{*}{ Order } & \multicolumn{4}{|c|}{$\mathbf{E}$} & \multicolumn{4}{|c|}{ I } \\
\hline & & \multicolumn{2}{|c|}{ Same } & \multicolumn{2}{|c|}{ Different } & \multicolumn{2}{|c|}{ Same } & \multicolumn{2}{|c|}{ Different } \\
\hline & & RTs & Errors & RTs & Errors & RTs & Errors & RTs & Errors \\
\hline Unexpected & $\begin{array}{l}\text { E first } \\
\text { I first }\end{array}$ & $\begin{array}{l}458 \\
569\end{array}$ & $\begin{array}{l}.000 \\
.000\end{array}$ & $\begin{array}{l}406 \\
508\end{array}$ & $\begin{array}{l}.000 \\
.067\end{array}$ & $\begin{array}{l}904 \\
561\end{array}$ & $\begin{array}{l}.300 \\
.200\end{array}$ & $\begin{array}{l}498 \\
415\end{array}$ & $\begin{array}{l}.000 \\
.000\end{array}$ \\
\hline Block & $\begin{array}{l}\text { E first } \\
\text { I first }\end{array}$ & $\begin{array}{l}605 \\
500\end{array}$ & $\begin{array}{l}.075 \\
.012\end{array}$ & $\begin{array}{l}456 \\
450\end{array}$ & $\begin{array}{l}.030 \\
.009\end{array}$ & $\begin{array}{l}502 \\
662\end{array}$ & $\begin{array}{l}.082 \\
.075\end{array}$ & $\begin{array}{l}388 \\
491\end{array}$ & $\begin{array}{l}.018 \\
.055\end{array}$ \\
\hline Mean & & 533 & .022 & 455 & .027 & 657 & .164 & 448 & .018 \\
\hline
\end{tabular}

Note $-E=$ equally spaced stimuli, $I=$ irregular stimuli, $E$ first $=$ equally spaced stimuli in the blocks first, I first = irregular stimuli in the blocks first. The error proportions are for responses to same and different stimuli. 
stimuli resulting in the FSSD pattern, as predicted. Simple effects comparisons indicated that the equally spaced stimuli resulted in significantly faster "same" responses and slightly slower "different" responses than did the irregular stimuli $[F(1,16)=16.23, p<.01$ and $F(1,16)$ $=.05$, respectively]. The response $\times$ stimulus $\times$ trial interaction was also significant $[F(1,16)=4.84$, $p<.05]$. It occurred because the difference between the "same" responses to equally spaced and irregular stimuli on the unexpected trials was greater than the difference between the "different" responses to equally spaced and irregular stimuli on the unexpected trials. Therefore, the FSSD pattern occurred for both the unexpected and block trials. Besides, this interaction did not occur in Experiments 2 and 3.

Two significant main effects also occurred. The responses were faster to equally spaced (strong whole) stimuli than to irregular (weak whole) stimuli $[F(1,16)$ $=7.29, p<.05]$, as predicted. "Same" responses were slower than "different" responses $[F(1,16)=9.66$, $p<.01]$. However, this latter result was not significant in Experiments 2 and 3.

The order $\times$ stimulus $\times$ trial interaction was highly significant $[F(1,16)=39.95, p<.001]$. This interaction is presumably a practice effect, because all four Stage1 means were higher than all four Stage-2 means. The four-way interaction was also significant $[F(1,16)=7.03$, $p<.05]$. It occurred because the decrease in RT from Stage 1 to Stage 2 was greater for the "same" responses than for the "different" responses.

The stimulus $\times$ trial, the order $\times$ trial, and the response $x$ stimulus $x$ trial interactions were also all significant ( $p<.05$ for each interaction). However, none of these interactions were significant in the corresponding ANOVAs for Experiments 2 and 3, and therefore they will not be considered.

Errors. In summing errors, all the responses to stimuli that immediately followed the unexpected trials were disregarded. The resulting error proportions are indicated in Table 1, which also indicates the mean proportions for the response $\times$ stimulus conditions. Note that these means are unweighted: the number of unexpected trials was much less than the number of block trials.

Errors were infrequent for most conditions. Therefore, for statistical analysis, errors were summed across the levels of the order and trial factors. The equally spaced (strong whole) stimuli resulted in a lower proportion of incorrect "different" response errors (i.e., errors to same stimuli) than did the irregular (weak whole) stimuli, although not significantly so ( $p>.10$, two-tailed, Wilcoxon signed ranks test). In addition, incorrect "same" response errors (i.e., errors to different stimuli) occurred with about equal frequency for the two types of stimuli.

\section{Discussion}

The equally spaced (strong whole) stimuli resulted in the FSSD pattern, in agreement with a majority of the studies mentioned earlier. In addition, the strong whole stimuli resulted in this pattern on both the unexpected and block trials. The unexpected trials were immediately preceded by block trials with the opposite type of stimulus. Therefore, the FSSD result was due to current stimuli, rather than to memory for preceding stimuli. In addition, it was not due to a difference in decision criteria between blocks of trials with the strong whole stimuli and blocks of trials with the weak whole stimuli. Furthermore, it was not due to a bias to respond fast to the equally spaced same stimuli, because the errors to these stimuli were less than the errors to the irregular same stimuli.

In the King and Atef-vahid (1986) and present experiments, the third object was extreme in size and in spatial position, respectively. Despite this correspondence, it was the weak whole stimuli that resulted in the FSSD pattern in the 1986 experiment. The instructions should have directed attention to the two small disks in the 1986 experiment and to all three disks in the present experiment. Discussion in the introduction therefore suggests that strong phenomenal wholes were associated with the FSSD pattern in both experiments. Features do not affect the type of attention under consideration. Consequently, a factor related to phenomenal wholes, rather than to features, may be the direct cause of the FSSD pattern.

Line-like strong wholes result in the FSSD pattern both when they contain more numerous parts (Beller, 1970) and when they contain more regular parts (the present experiment). Therefore, these two results indicate that a particular type of feature is not required for line-like phenomenal wholes to be associated with the FSSD pattern.

The strong whole stimuli also resulted in significantly faster overall ("same" plus "different") responses than did the weak whole stimuli. As for the FSSD result, the block and unexpected trials procedure indicates that the obtained result was due to current stimuli, and that it was not due to a criterion shift. The strong whole stimuli also resulted in fewer overall errors than did the weak whole stimuli. Therefore, the strong whole stimuli resulted in superior overall performance.

\section{EXPERIMENT 2}

In Experiment 1, the disks of the equally spaced and irregular stimuli differed in spatial position, and the different stimuli differed in size. The latter difference also entails a difference in spatial position: the edges of the disks that differed in size also differed in spatial position. Therefore, the obtained results might be of limited generality. Parts that differ in color need not entail any difference in spatial position. Therefore, Experiment 2 essentially replicated Experiment 1, but with different stimuli that were different in achromatic color. A second purpose was to employ a complete set of stimuli.

\section{Method}

Open circles that were $5 \mathrm{~mm}$ in diameter were obtained from Letraset Sheet 2453. These circles were filled in with Berol Verithin Black or Light Grey pencils. 
The equally spaced (strong whole) stimuli included $B-B-B$, G-G-G, B-G-B, B-G-G, G-B-B, and G-B-G stimuli, and the irregular (weak whole) stimuli included BB- -B, B--BB, GG--G, G--GG, B--GB, BG--G, GB--B, and G--BG stimuli. B indicates a black disk, and $\mathrm{G}$ indicates a gray disk. A B-B-B stimulus is an equally spaced stimulus, a BB--B stimulus is an irregular stimulus in which the two close circles are to the left, and so on.

The remainder of the method was the same as for Experiment 1, except for the obvious difference that subjects were instructed to respond "same" or "different" depending on the color of the disks.

\section{Results}

Response times. The entries for the ANOVA were obtained essentially as before. However, the larger number of stimuli led to slight modifications. Specifically, the RTs to both the all-black and all-gray stimuli were summed to obtain the "same" response entries, and the RTs to the two black and one gray and one black and two gray stimuli were summed to obtain the "different" response entries.

The same ANOVA was used. Table 2 shows the mean RTs and error proportions for the 16 conditions. The interaction between the response and stimulus factors was again significant $[F(1,16)=12.59, p<.01]$. The response $\times$ stimulus interaction means in Table 1 reveal that the equally spaced (strong whole) stimuli resulted in significantly faster "same" responses and nonsignificantly slower "different" responses than the irregular (weak whole) stimuli $[F(1,16)=11.63, p<.01$ and $F(1,16)$ $=2.56, p>.10$, respectively]. Thus, Experiment 2 replicated the Experiment 1 finding that the strong whole stimuli resulted in the FSSD pattern.

The equally spaced stimuli again resulted in faster overall ("same" plus "different") responses than did the irregular stimuli, although not significantly so $[F(1,16)=$ $1.64, p>.10]$.

The following significant and marginally significant interactions occurred: the response $\times$ stimulus $\times$ trial interaction $[F(1,16)=4.13, p<.10]$, the order $\times$ stimulus $\times$ trial interaction $[F(1,16)=8.98, p<.01]$, and the four-way interaction $[F(1,16)=3.49, p<.10]$. They all resemble the corresponding interactions of Experiment 1.

Errors. The errors were calculated as in Experiment 1. The equally spaced (strong whole) stimuli again resulted in fewer incorrect "different" response errors (i.e, errors to same stimuli) than did the irregular stimuli, although once more not significantly so $(p>.10$, twotailed, Wilcoxon signed ranks test). In addition, the two types of stimuli again resulted in about the same frequency of incorrect "same" response errors (i.e., errors to different stimuli).

\section{Discussion}

Experiment 2 replicated the results of Experiment 1. Therefore, Experiment 2 indicates that the difference in the spatial position of the three disks was influential, even though their color varied independently of spatial position. Hawkins and Shigley (1972) also obtained these FSSD and overall performance results with different stimuli that differed in color.

\section{EXPERIMENT 3}

One purpose of Experiment 3 was to determine whether an additional type of a difference in features would again result in an association between strong phenomenal wholes and the FSSD and superior overall performance results. The presence or absence of a gap does not closely involve previously considered similarities in size, shape, color, the regularity of spacing, symmetry, linearity, and parallelness (see the introduction). Therefore, a gap was inserted between the parts of a strong whole stimulus to result in a weak whole stimulus. The traditional outgoingfins variation of the Müller-Lyer illusion was used, and the gap appeared between the fins and the ends of the center line (see Figure 2).

The gap results in an increase in the number of discrete objects that are present (from one to three), and this increase results in a weaker phenomenal whole. In contrast, an increase in the number of discrete objects that were present in the previously considered research resulted in stronger phenomenal wholes (see the introduction). Therefore, the presence or absence of a gap would indicate whether a larger number of discrete objects or a stronger phenomenal whole is more consistently associated with the FSSD pattern.

Another purpose of Experiment 3 was to employ less ambiguous strong and weak whole stimuli than in Experiments 1 and 2. The equally spaced stimuli of Experiments 1 and 2 can be apprehended as three separate disks (wholes), in addition to being apprehended as a single line-

Table 2

Mean RTs and Errors for Experiment 2

\begin{tabular}{|c|c|c|c|c|c|c|c|c|c|}
\hline \multirow[b]{3}{*}{ Trial } & \multirow[b]{3}{*}{ Order } & \multicolumn{4}{|c|}{ E } & \multicolumn{4}{|c|}{ I } \\
\hline & & \multicolumn{2}{|c|}{ Same } & \multicolumn{2}{|c|}{ Different } & \multicolumn{2}{|c|}{ Same } & \multicolumn{2}{|c|}{ Different } \\
\hline & & RTs & Errors & RTs & Errors & RTs & Errors & RTs & Errors \\
\hline Unexpected & $\begin{array}{l}\text { E first } \\
\text { I first }\end{array}$ & $\begin{array}{l}453 \\
497\end{array}$ & $\begin{array}{l}.000 \\
.100\end{array}$ & $\begin{array}{l}457 \\
457\end{array}$ & $\begin{array}{l}.000 \\
.067\end{array}$ & $\begin{array}{l}608 \\
502\end{array}$ & $\begin{array}{l}.400 \\
.200\end{array}$ & $\begin{array}{l}417 \\
421\end{array}$ & $\begin{array}{l}.000 \\
.000\end{array}$ \\
\hline Block & $\begin{array}{l}\text { E first } \\
\text { I first }\end{array}$ & $\begin{array}{l}469 \\
450\end{array}$ & $\begin{array}{l}.050 \\
.000\end{array}$ & $\begin{array}{l}452 \\
423\end{array}$ & $\begin{array}{l}.010 \\
.036\end{array}$ & $\begin{array}{l}438 \\
506\end{array}$ & $\begin{array}{l}.035 \\
.025\end{array}$ & $\begin{array}{l}417 \\
451\end{array}$ & $\begin{array}{l}.018 \\
.050\end{array}$ \\
\hline Mean & & 467 & .038 & 447 & .028 & 514 & .165 & 427 & .017 \\
\hline
\end{tabular}

Note-E = equally spaced stimuli, I = irregular stimuli, E first = equally spaced stimuli in the blocks first, I first = irregular stimuli in the blocks first. The error proportions are for responses to same and different stimuli. 


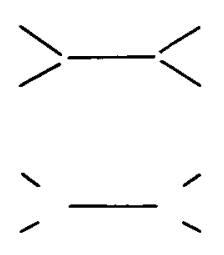

Figure 2. The traditional and gap variations of the Müler-Lyer illusion for Experiment 3.

like whole. In contrast, the traditional variation of the Müller-Lyer illusion seems to be consistently apprehended as a single event (whole) that roughly approximates a bow tie: it is nearly impossible to apprehend it as three separate parts to a greater extent than the gap variation. Therefore, the traditional variation forms an obviously stronger single whole than the gap variation.

The last purpose of Experiment 3 involved the finding that the traditional variation of the Müller-Lyer illusion results in a greater overestimation of the length of the center line than does the gap variation (Coren \& Girgus, 1972). The assimilation theory of the Müller-Lyer illusion (Krueger, 1972; Pressey, Di Lollo, \& Tait, 1977) assumes that the center line of this illusion is perceived as similar to (assimilates with) the large distance between the outgoing fins, thereby accounting for the overestimation of the center line. The FSSD pattern is considered to indicate high perceived similarity (see the introduction). Therefore, if the traditional variation resulted in the FSSD pattern, it would provide independent evidence that the center line assimilates with the distance between the outgoing fins.

\section{Method}

For same stimuli, the fins and the center line of both the traditional and gap stimuli were either both red or both green. For different stimuli, the fins were red and the center line was green, or the reverse. Thus, there were eight stimuli in all.

For both the traditional and gap stimuli, the length of the center line was $20 \mathrm{~mm}$, and the horizontal length of the total stimulus was $41 \mathrm{~mm}$. For the traditional stimuli, the length of the fin lines was $11 \mathrm{~mm}$ in diagonal extent. In addition, there were 1-mm gaps between the ends of the center line and the beginning of the fin lines in order to avoid an overlap of the two colors during the preparation of the stimuli. The fins for the gap stimuli were identical, except that the gaps between the ends of the center line and the be- ginning of the fin lines were $8 \mathrm{~mm}$. Berol Verithin Scarlet Red and Grass Green pencils were used to draw the stimuli.

The method was the same as for Experiment 2, except that the blocks of each stage occurred in a new random order, and there were 6 subjects in each of the independent groups.

\section{Results}

The ANOVA was the same as for Experiments 1 and 2 , except that the traditional and gap stimuli were the strong and weak whole stimuli, respectively. Scores for the ANOVA were obtained in essentially the same way. as for Experiment 2.

Table 3 indicates the mean RTs and error proportions for the 16 experimental conditions. The interaction between the response and stimulus factors was again significant $[F(1,20)=28.11, p<.001]$. The interaction means in Table 3 reveal that the traditional (i.e., strong whole) stimuli again resulted in the FSSD pattern. In addition, the strong whole stimuli resulted in significantly faster "same" responses and nonsignificantly slower "different" responses than did the gap (i.e., weak whole) stimuli $[F(1,20)=34.64, p<.001$ and $F(1,20)=2.60$, $p>.10$, respectively].

The strong whole stimuli again resulted in faster overall ("same" plus "different") responses than did the weak whole stimuli $[F(1,20)=9.12, p<.01]$.

The following obtained significant or marginally significant interactions closely resemble those of Experiments 1 and 2: the response $\times$ stimulus $\times$ trial interaction $[F(1,20)=3.36, p<.10]$, the order $\times$ stimuli $\times$ trial interaction $[F(1,20)=15.93, p<.001]$, and the four-way interaction $[F(1,20)=11.76, p<.01]$.

Errors. The errors were summed as in the previous experiments. The traditional (i.e., strong whole) stimuli resulted in significantly fewer incorrect "different" responses (i.e., errors to same stimuli) than did the gap (i.e., weak whole) stimuli ( $p<.05$, two-tailed, Wilcoxon signed ranks test). The strong whole stimuli also resulted in negligibly fewer incorrect "same" responses (i.e., errors to different stimuli) than did the weak whole stimuli.

\section{Discussion}

The results were very similar to those of Experiments 1 and 2. Therefore, a different type of feature, the presence or absence of a gap, led to an association between strong

Table 3

Mean RTs and Errors for Experiment 3

\begin{tabular}{|c|c|c|c|c|c|c|c|c|c|}
\hline \multirow[b]{3}{*}{ Trial } & \multirow[b]{3}{*}{ Order } & \multicolumn{4}{|c|}{$\mathrm{T}$} & \multicolumn{4}{|c|}{$\mathbf{G}$} \\
\hline & & \multicolumn{2}{|c|}{ Same } & \multicolumn{2}{|c|}{ Different } & \multicolumn{2}{|c|}{ Same } & \multicolumn{2}{|c|}{ Different } \\
\hline & & RTs & Errors & RTs & Errors & RTs & Errors & RTs & Errors \\
\hline Unexpected & $\begin{array}{l}T \text { first } \\
G \text { first }\end{array}$ & $\begin{array}{l}416 \\
514\end{array}$ & $\begin{array}{l}.083 \\
.000\end{array}$ & $\begin{array}{l}478 \\
499\end{array}$ & $\begin{array}{l}.056 \\
.000\end{array}$ & $\begin{array}{l}575 \\
544\end{array}$ & $\begin{array}{l}.333 \\
.000\end{array}$ & $\begin{array}{l}431 \\
443\end{array}$ & $\begin{array}{l}.056 \\
.000\end{array}$ \\
\hline Block & $\begin{array}{l}T \text { first } \\
G \text { first }\end{array}$ & $\begin{array}{l}480 \\
430\end{array}$ & $\begin{array}{l}.042 \\
.029\end{array}$ & $\begin{array}{l}420 \\
482\end{array}$ & $\begin{array}{l}.032 \\
.044\end{array}$ & $\begin{array}{l}471 \\
591\end{array}$ & $\begin{array}{l}.056 \\
.069\end{array}$ & $\begin{array}{l}418 \\
493\end{array}$ & $\begin{array}{l}.018 \\
.071\end{array}$ \\
\hline Mean & & 460 & .039 & 470 & .033 & 545 & .115 & 446 & .036 \\
\hline
\end{tabular}

Note- $\mathrm{T}=$ traditional stimuli, $\mathrm{G}=$ gap stimuli, $\mathrm{T}$ first $=$ traditional stimuli in the blocks first, $\mathrm{G}$ first = gap stimuli in the blocks first. The error proportions are for responses to same and different stimuli. 
phenomenal wholes and the FSSD and superior overall performance results.

The gap (weak whole) stimuli did not result in the FSSD pattern, but they did contain more discrete objects than the traditional (strong whole) stimuli. In contrast, in other studies (e.g., Beller, 1970), the stimuli with more discrete objects resulted in stronger phenomenal wholes and the FSSD pattern. Therefore, strong phenomenal wholes are more consistently associated with the FSSD pattern than are a large number of discrete objects.

The three equally spaced (strong whole) disk stimuli of Experiments 1 and 2 also resulted in the FSSD pattern and superior overall performance. Therefore, the present experiment counters the possibility that the three equally spaced disk stimuli of Experiments 1 and 2 functioned as three separate objects (wholes).

The FSSD pattern can be considered to indicate a high perceived similarity among parts. Therefore, the present FSSD finding also backs the assumption that the center line of the Müller-Lyer illusion is overestimated because it assimilates with the large distance between the fins.

Fox (1975) employed pairs of letters that overlapped to a small extent or were separated by a gap (as is typical). Nevertheless, the overlapping letters resulted in neither the FSSD pattern nor superior overall performance. The overlap made the letters resemble embedded figures, whereas embeddedness was less extensive in the present experiment.

\section{GENERAL DISCUSSION}

\section{Phenomenal Wholes, Features, and the FSSD Pattern}

The introduction indicated that strong phenomenal wholes are usually associated with the FSSD pattern. The three present experiments also obtained this result.

Are strong phenomenal wholes more closely related to the FSSD pattern than is a particular type of feature? The introduction indicated that stimuli with more physically similar parts and stimuli that consist of a larger number of objects, result in strong phenomenal wholes and the FSSD pattern. The present Experiments 1 and 2 support the same point for specifically line-like phenomenal wholes. This is because line-like phenomenal wholes were associated with the FSSD pattern when spacing was varied (Experiments 1 and 2) and also when the number of parts was varied (the Beller, 1970, result). The present research also found that strong phenomenal wholes were associated with the FSSD pattern when the weak whole stimuli were formed by inserting a gap between the parts of the strong whole stimuli. This result is of interest because the gap condition did not closely involve the types of similarity that were previously investigated. Furthermore, the gap increased the number of discrete objects that were present from one to three, whereas in previous research, an increase in this number resulted in the FSSD pattern. Therefore, the strength of phenomenal wholes is more closely related to the FSSD pattern than is the number of discrete objects that are present. In addition, FSSD evidence was obtained even though color varied independently of spatial position. Also, the overall size of the display and the average distance between parts were not factors in both the present and earlier research.

A comparison between King and Atef-vahid's Experiment 5 (1986) and the present Experiments 1 and 2 led to the possibility that attention can determine which parts of the total display are experienced as a whole, and that the attended-to stronger phenomenal whole is associated with the FSSD pattern. This possible influence of attention also suggests that phenomenal wholes are more closely related to the FSSD pattern than are features.

\section{The Whole-Assimilation Position}

The introduction reasoned that the FSSD pattern indicates that the perceived similarity among parts is high. Stimuli that result in strong phenomenal wholes result in this pattern. Therefore, strong phenomenal wholes have parts that are perceived as more similar than do weak phenomenal wholes.

This whole-assimilation position is supported by research that did not employ the "same"-"different" response task (King, in press). The Koffka ring figure (Koffka, 1935) is illustrative: the left and right gray parts are perceived as more similar when they belong to the continuous ring-the stronger phenomenal whole. In addition, the perception of the spread of color tends to be confined to parts of the same phenomenal whole (e.g., Day, 1983). Also, parts of the same whole are perceived to be in more similar spatial positions (Coren \& Girgus, 1980). Furthermore, a number of illusions-including the Müller-Lyer, Baldwin, divided line, Ponzo, Delboeuf, Ebbinghaus, and parallel line illusions, and also the small angle variation of the Zoellner illusion - can all be considered to be assimilation outcomes that result from parts belonging to a single phenomenal whole. Moreover, Experiment 3 supports the assimilation interpretation of the Müller-Lyer illusion, as was noted.

Anchor (extreme) stimuli might increase the perceived similarity of similar stimuli (King, 1983; King \& Atefvahid, 1986). They might also increase the extent to which these stimuli group, much as an extreme third object appears to make two more positionally similar objects group more strongly. Therefore, the anchor effect might also be a whole-assimilation outcome.

\section{Phenomenal Wholes and Overall \\ "Same"-"Different" Performance}

According to the introduction, previous studies indicate that strong phenomenal wholes are frequently associated with superior overall ("same" plus "different") performance, according to RT and error evidence. The three present experiments also obtained this outcome. Furthermore, Experiment 3 employed a gap to form the weak whole stimuli, which means that an increase in similarity in parts is not required for strong wholes to be associated with superior overall performance. 


\section{Phenomenal Wholes and Identification \\ Task Performance}

The "same"'-"different" task can be considered to require the identification (and then the comparison) of two simultaneously occurring targets. Performance on the divided-attention type of identification task improves when the two targets belong to the same phenomenal whole (Duncan, 1984). Furthermore, full and partial report (Fryklund, 1975; Kahneman \& Henik, 1977) and speeded response results (Pollatsek \& Digman, 1977, Experiment 6) also indicate that identification improves when multiple simultaneously occurring targets belong to the same phenomenal whole. Therefore, both "same"-" different" and identification task evidence indicate that the ability to identify multiple targets frequently improves when they belong to the same phenomenal whole.

\section{The Perceived Similarity of Parts and Their Identification}

Taken together, the whole-assimilation evidence and the evidence involving phenomenal wholes and the identification of multiple targets implies that the perception of parts as similar is positively associated with the ability to identify multiple parts. A related result is that a prime improves the identification of a similar target. However, if parts are perceived as too similar, the resulting difficulty in discriminating between them should counteract the priming-like advantage and, therefore, identification should suffer. Consequently, strong phenomenal wholes should not always be associated with superior overall performance, which provides an explanation of why strong phenomenal wholes are more consistently associated with the FSSD pattern than with superior overall "same"-"different" performance (see the introduction).

In conclusion, the present results replicated earlier FSSD and superior overall performance results using stimuli selected so as to form strong and weak phenomenal wholes. In addition, evidence was obtained showing that phenomenal wholes are more closely related to the FSSD pattern than are features. Also, the FSSD result supports the whole-assimilation position, and the superior overall performance result supports the position that the identification of multiple parts frequently improves when they belong to the same phenomenal whole.

\section{REFERENCES}

Beller, H. K. (1970). Parallel and serial stages in matching. Journal of Experimental Psychology, 84, 213-219.

Chignell, M. H., \& Krueger, L. E. (1984). Further evidence for priming in perceptual matching: Temporal, not spatial, separation enhances the fast-same effect. Perception \& Psychophysics, 36, 257-265.

Coren, S., \& Girgus, J. S. (1972). Differentiation and decrement in the Mueller-Lyer illusion. Perception \& Psychophysics, 12, 466-470. Coren, S., \& Girgus, J. S. (1980). Principles of perceptual organiza- tion and spatial distortion: The gestalt illusions. Joumal of Experimental Psychology: Human Perception \& Performance, 6, 404-412.

DAY, R. H. (1983). Neon color spreading, partially delineated borders, and the formation of illusory contours. Perception \& Psychophysics, 34, 488-490.

Dixon, P., \& Just, M. A. (1978). Normalization of irrelevant dimensions in stimulus comparisons. Journal of Experimental Psychology: Human Perception \& Performance, 4, 36-46.

DonderI, D. C., \& Zelnicker, D. (1969). Parallel processing in visual same-different decisions. Perception \& Psychophysics, 5, 197-200.

DUNCAN, J. (1984). Selective attention and the organization of visual information. Journal of Experimental Psychology: General, 113, 501-517.

Egeth, H. E., Brownell, H. H., \& Geoffrion, L. D. (1976). Testing the role of vertical symmetry in letter matching. Journal of Experimental Psychology: Human Perception \& Performance, 2, 429-434.

Fox, J. (1975). The use of structural diagnostics in recognition. Journal of Experimental Psychology: Human Perception \& Performance, 1, 57-67.

FrYKLUND, I. (1975). Effects of cued-set spatial arrangement and targetbackground similarity in the partial-report paradigm. Perception \& Psychophysics, 17, 375-386.

Gravetter, F., \& Lockhead, G. R. (1973). Criterial range as a frame of reference for stimulus judgment. Psychological Review, 80, 203-216.

HAWkiNs, H. L., \& SHigley, R. H. (1972). Irrelevant information and processing mode in speeded discrimination. Journal of Experimental Psychology, 96, 389-395.

Kahneman, D., \& HeniK, A. (1977). Effects of visual grouping on immediate recall and selective attention. In S. Dornic (Ed.), Attention and performance VI (pp. 307-322). Hillsdale, NJ: Erlbaum.

KING, D. L. (1983). Anchor research: Evidence for an increase in the perceived similarity of stimuli obtained with a speeded response paradigm. Journal of Experimental Psychology: Human Perception \& Performance, 9, 474-486.

KING, D. L. (in press). Assimilation is due to one perceived whole and contrast is due to two perceived wholes. New ldeas in Psychology.

KING, D. L., \& ATEF-VAHID, M. (1983). Two extensions of the anchorrange effect. Perception \& Psychophysics, 39, 96-104.

KoffKA, K. (1935). Principles of gestalt psychology. New York: Harcourt.

KRUEGER, L. E. (1972). Gregory's theory of illusions: Some disconfirming evidence in the case of the Müller-Lyer illusion. Psychological Review, 79, 538-539.

KRUEGER, L. E. (1973). Effect of irrelevant surrounding material on speed of same-different judgment of two adjacent letters. Journal of Experimental Psychology, 98, 252-259.

KrUEGER, L. E., \& Shapiro, R. G. (1981). Intertrial effects of samedifferent judgments. Quarterly Journal of Experimental Psychology, 33A, 241-265.

Millspaugh, J. R. (1978). Effects of array organization on samedifferent judgments. Perception \& Psychophysics, 23, 27-35.

Pollack, I. (1952). The information of elementary auditory displays. Journal of the Acoustical Society of America, 24, 745-749.

Pollatsek, A., \& Digman, L. (1977). Dependent spatial channels in visual processing. Cognitive Psychology, 9, 326-352.

Pressey, A. W., Di Lollo, V., \& TAIt, R. W. (1977). Effects of gap size between shaft and fins and of angle of fins on the Müller-Lyer illusion. Perception, 6, 435-439.

RichARDS, J. T. (1978). Interitem structure and the facilitation of simultaneous comparison. Journal of Experimental Psychology: Human Perception \& Performance, 4, 72-87.

(Manuscript received May 11, 1987; revision accepted for publication November 2, 1987.) 